
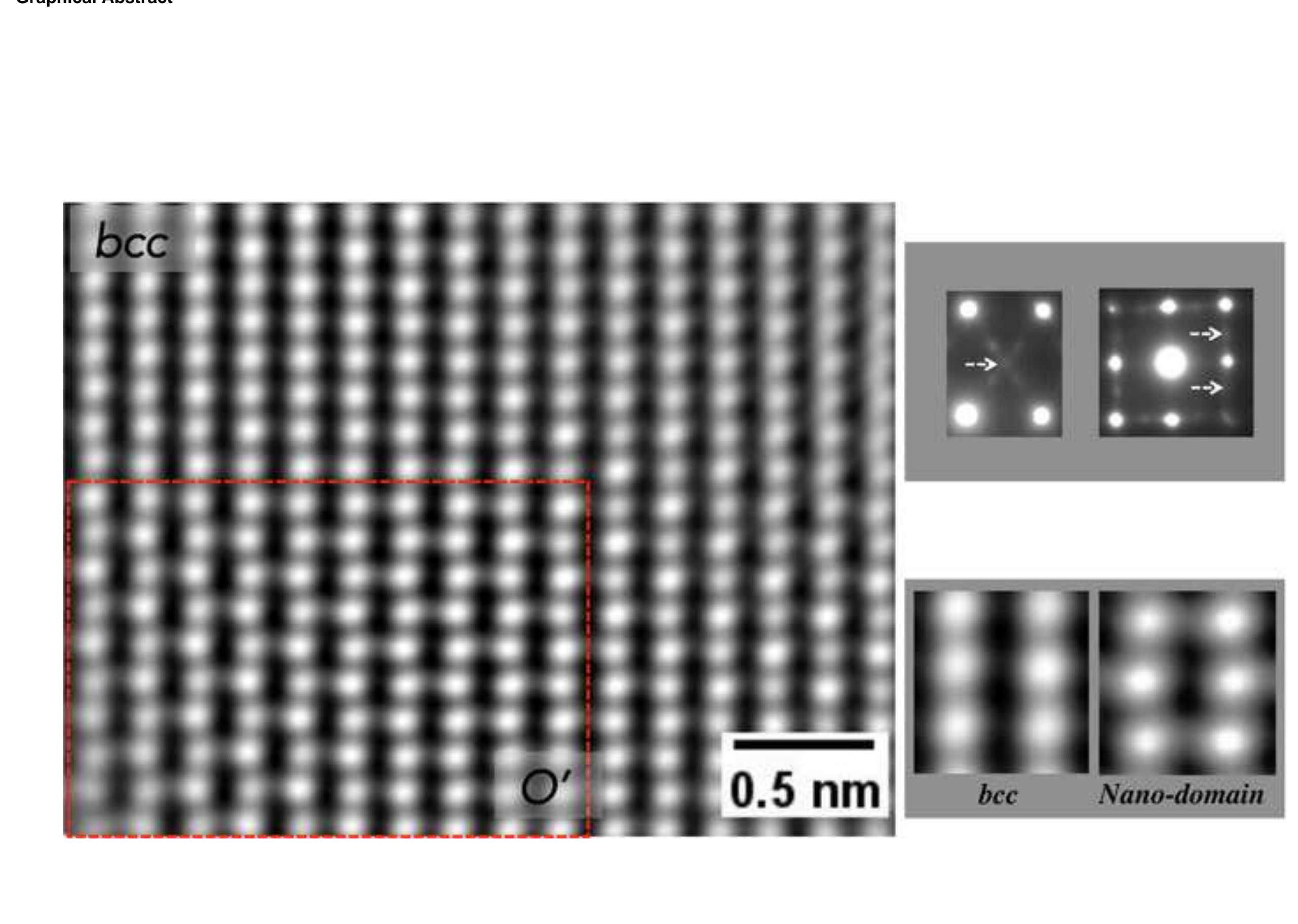


\title{
The Effect of Alloy Composition on Instabilities in the $\beta$ Phase of Titanium Alloys
}

Yufeng Zheng ${ }^{1}$, Robert E.A. Williams ${ }^{1}$, Soumya $\mathrm{Nag}^{2}$, Rajarshi Banerjee ${ }^{3}$, Hamish L. Fraser $^{1, *}$ and Dipankar Banerjee ${ }^{4}$

${ }^{1}$ Center for the Accelerated Maturation of Materials and Department of Materials Science and Engineering, The Ohio State University, 1305 Kinnear Road, Columbus, $\mathrm{OH} 43212$, USA.

${ }^{2}$ General Electric Company, Global Research Center, Niskayuna, NY 12309 USA

${ }^{3}$ Center for Advanced Research and Technology and Department of Materials Science and Engineering, University of North Texas, 3940 N. Elm, Denton, TX 76207, USA

${ }^{4}$ Department of Materials Engineering, Indian Institute of Science, C V Raman Ave, Bengaluru, Karnataka 560012, India

"Corresponding author: fraser.3@osu.edu, CEMAS, The Ohio State University, 1305 Kinnear Rd., Suite 100, Columbus, OH 43212-1177, USA

\begin{abstract}
A metastable nano-scale disordered precipitate with orthorhombic symmetry has been identified using high resolution scanning transmission electron microscopy. The phase, termed $\mathrm{O}^{\prime}$, is metastable, formed by a shuffle mechanism involving a $\{110\}<1 \overline{1} 0>$ transverse phonon wave in samples of $\mathrm{Ti}-26 \mathrm{Nb}-2 \mathrm{Zr}$ (at\%) quenched from the $\beta$ phase. The addition of $2 \% \mathrm{Zr}$ to Ti-26Nb appears to suppress significantly the stability of both the $\{11 \overline{2}\}<111>$ shear and $2 / 3<111>$ longitudinal phonon wave but promotes the $\{110\}<1 \overline{1} 0>$ transverse shuffle. This results in the nano-size $\mathrm{O}^{\prime}$ phase being homogeneously formed in the parent $\beta$ phase matrix rather than the massive $\alpha^{\prime \prime}$ phase.
\end{abstract}

Keywords: Titanium alloys; Phase transformations; High-resolution electron microscopy; Martensite;

In titanium alloys, a variety of elastic instabilities have been associated with transformations from the $\beta$ phase to martensite ( $\alpha^{\prime}$ phase or $\alpha^{\prime \prime}$ phase) and the $\omega$ phase, as shown in Fig.1. The martensite transformation to the $\alpha^{\prime}$ phase with the hcp crystal structure is related to the $\{11 \overline{2}\}<111>$ shear that transforms the $\{110\}_{\beta}$ planes into the close packed planes of the hexagonal martensite while a $\{110\}<1 \overline{1} 0>$ displacement wave leading to a shuffle on every alternate plane changes the stacking sequence of these close packed planes, as proposed by Burgers in 1934 [1]. The orthorhombic martensite ( $\alpha$ " phase) represents a smaller magnitude of these two shears so that the resulting structure has an orthorhombic, rather than hexagonal, symmetry. The Burgers model assumes that the $\{11 \overline{2}\}<111>$ shear occurs first followed by $\{110\}<1 \overline{1} 0>$ shuffle and this transformation is described in Fig. 1(a). 
As has been pointed out, there is no reason why the softening modes cannot be reversed [2]. If the $\{110\}<1 \overline{1} 0>$ displacement wave occurs first, Fig 1(b), twelve variants of these shears can result, in opposite directions for each of the six $<110>$ vectors. The formation of nano-domains of this kind has recently been suggested to occur as a consequence of oxygen addition to titanium alloys in alloys bounding the domain of compositions relating to gum metal $[3,4]$. The occurrence of $\{110\}<1 \overline{10}>$ shears has been deduced from the presence of $<110>$ relrods in reciprocal space [3, 4]. It has been suggested that the oxygen atoms are located at the octahedral interstitial sites and can produce local strain, and therefore suppress the long-range martensite transformation.

A third elastic instability involves the $\omega$ phase formation. This is related to the longitudinal $2 / 3<111>$ phonon wave vector and is shown in Fig. 1(c). It is well known that with increasing $\beta$ alloying content a transition from orthorhombic martensite $\left(\alpha^{\prime \prime}\right)$ to athermal $\omega$ occurs in quenched $\beta$ alloys of titanium [5]. Two adjacent $\{111\}$ atom planes of every three in $b c c$ matrix will collapse into one leaving the third unaltered and transform the $b c c$ structure of $\beta$ phase to the hexagonal structure of $\omega$ phase [6, 7].

The aim of the present research is firstly to explore the effect of alloying with substitutional solute on the elastic instabilities related to the $\{110\}<1 \overline{1} 0>$ shuffle and the $\omega$ and martensitic transformations, and secondly to image directly the structure of the nano-domains formed by the $\{110\}<1 \overline{10}>$ shuffle. The choice of compositions for this study was guided by the anomalous effect of $\mathrm{O}, \mathrm{Al}, \mathrm{Zr}$ and $\mathrm{Sn}$ on the stability of $\omega$ and martensite in $\beta$ stabilized alloys at the limit of stability of the $\beta$ phase. Thus, it is known that these elements, which are otherwise $\alpha$ stabilizers or neutral elements, suppress the formation of the $\omega$ phase and martensite [8]. Specifically in this note, the effect of $\mathrm{Zr}$ and $\mathrm{Al}$ on the these two instabilities in $\mathrm{Ti}-\mathrm{Nb}$ and Ti-Mo alloys, respectively, is explored, and the first conclusive evidence for a structure that is developed by the operation of the $\{110\}<1 \overline{1} 0>$ shuffle on every alternate $\{110\}$ plane of the parent $\beta$ phase is provided.

The choice of alloy compositions were determined by a consideration of the Bo-Md diagram proposed by Abdel-Hady et al [9] to describe the stability of the $\beta$ phase. The binary compositions $\mathrm{Ti}-26 \mathrm{Nb}$ and Ti-9Mo lie adjacent to the $\beta / \beta+\omega$ boundary (compositions in atomic \%). The addition of $2 \% \mathrm{Zr}$ to a Ti-26Nb alloy and $9 \% \mathrm{Al}$ to a Ti-9Mo change the location of the alloys on the Bo-Md diagram such that martensite would be expected to form on quenching the $\beta$ phase (see Figure 1 of supplementary figures). Hence, the alloys $\mathrm{Ti}-26 \mathrm{Nb}$ and $\mathrm{Ti}-26 \mathrm{Nb}-2 \mathrm{Zr}$ were melted by non consumable vacuum arc melting, re-melted 4 times and homogenized at $1000^{\circ} \mathrm{C}$ for 4 days before $\beta$ solution at $1000^{\circ} \mathrm{C}$ for 30 minutes followed by a water quench. Ti-9Mo and Ti9Mo-9Al were provided by TIMETAL Corporation and the as-received alloys were homogenized in vacuum furnace at $1100^{\circ} \mathrm{C}$ for 96 hours. $\beta$ solution treatment selected for Ti-9Mo was $1000^{\circ} \mathrm{C}$ for 30 minutes (the same as used in [6]), and Ti-9Mo-9Al $910^{\circ} \mathrm{C}$ for 1 hour $\left(100^{\circ} \mathrm{C}\right.$ higher than calculated $\mathrm{T}_{\beta}$ using Thermo-Calc). Site-specific TEM foils were prepared using the Dual Beam Focused Ion Beam (DB-FIB) technique, specifically a FEI Helios Nanolab 600 system, and ion beam damage on the foil surfaces was minimized using low voltage $\mathrm{Ar}^{+}$ion milling using a Fischione 
Nanomill ${ }^{\mathrm{TM}}$ Model 1040. TEM dark-field images and diffraction patterns were recorded on a Philips CM200 transmission electron microscope operating at an accelerating voltage of $200 \mathrm{kV}$ and HAADF-STEM images were recorded using a probe-corrected FEI Titan ${ }^{3} \mathrm{TM}$ 80-300 operated at $300 \mathrm{kV}$, using a convergence semiangle of $12 \mathrm{mrad}$ and a collection semi-angle of $44 \mathrm{mrad}$, with an incident beam dwell-time of $4 \mu \mathrm{s} / \mathrm{px}$ and a beam current of $\approx 90 \mathrm{pA}$.

Figure 2 shows diffraction patterns from $\beta$ quenched Ti-26Nb (Fig. 2(a)) and Ti26Nb-2Zr (Fig. 2(b)), and Ti-9Mo and Ti-9Mo-9Al (Fig. 2(c and d)). In the $<110>_{\beta}$ zone axis, Fig. 2 (a,b,c and d), $\omega$ phase reflections are present at $1 / 3$ and $2 / 3\{112\}_{\beta}$ locations (examples shown by red (solid) arrows in the figures). In addition, the patterns in these figures shows streaking along $<112>_{\beta}$ directions resulting in intensity maxima at $1 / 2<112>_{\beta}$ positions (examples shown by white (dashed) arrows in the figures). The addition of $\mathrm{Zr}$ to the binary $\mathrm{Ti}-\mathrm{Nb}$ alloy and $\mathrm{Al}$ to the binary Ti-Mo alloy, Fig. 2(b and d), results in a decrease of intensity of the $\omega$ reflections and an enhancement of the streaking and diffuse intensity maxima occurring at the $1 / 2\{110\}_{\beta}$. There is no evidence of martensite $\left(\alpha^{\prime \prime}\right)$ formation. Diffraction patterns were also recorded in the $\langle 100\rangle_{\beta}$ zone axis of Ti-26Nb-2Zr, shown in Fig. 2(e and f). Fig. 2(e) shows the on-zone axis pattern, in which streaking along $<110>_{\beta}$ directions is present along with intensity maxima in $1 / 2\{110\}_{\beta}$ (an example marked by a white arrow) and $1 / 2\{310\}_{\beta}$ positions (an example shown by the white circle in the figures). Tilting about the $\langle 110\rangle_{\beta}$ axis by small amounts eliminates the intensity at the $1 / 2$ $\{110\}_{\beta}$ positions as shown in Fig. 2(f), indicating that their origin lies in double diffraction. The indexing and interpretation of the patterns will be discussed below.

The dark-field image in Fig. 3(a) recorded from the Ti-26Nb-2Zr alloy using the intensity maxima in the $1 / 2(310)_{\beta}$ position (Fig. 2(f)) shows the presence of nanodomains similar to earlier observations [3, 4]. These nano-size domains were examined by HAADF-STEM imaging in an aberration corrected microscope, with the electron beam parallel to $[110]_{\beta}$. The fast Fourier transform (Fig. 3(b)) from the raw image shows spatial frequencies from both the $\omega$ phase (at $1 / 3$ and $2 / 3\{112\}_{\beta}$ ), highlighted by red solid circles in the figure, and at $1 / 2\{112\}_{\beta}$ positions, indicated by the blue dashed circle. A high magnification HAADF image is shown in Fig. 3(c), in which it is clear that in the lower left part of the image, bounded by the dashed red box, the $b c c$ symmetry has been broken by small displacements of every other atomic column. These displacements result in a structure very similar to that shown in the schematic diagram in Fig. 1(b), which depicts the result of the application of the $\{110\}<1 \overline{1} 0>$ shuffle, i.e., a disordered orthorhombic structure. Thus, it is apparent that a displacement of atoms from bcc positions in Fig. 3(c) has occurred and these displacements are identified unequivocally to be those expected from the presence of nano-domains formed by the shuffle of atoms in the $\langle 1 \overline{1} 0>$ direction on every alternate $\{110\}$ plane of $\beta$. Highly magnified images from the $b c c$ matrix and a nanodomain are compared in Fig. 3(d), where the displacements of the atomic columns in the domain may be distinguished readily.

To explain the diffraction maxima observed at $1 / 2(310)_{\beta}$ positions in the $\langle 100\rangle_{\beta}$ zone axis and the $1 / 2(112)_{\beta}$ positions in the $\langle 110\rangle_{\beta}$ zone axis a structure, termed the $\mathrm{O}^{\prime}$ phase, with $\mathrm{Cmcm}$ symmetry has been simulated in which the atoms on every alternate plane of the parent $\beta$ phase are displaced by a small fraction along the 
$<110>_{\beta}$ direction consistent with the operation of the $\{110\}<1 \overline{10}>$ shuffle. The aaxis of the structure coincides with the a-axis of the $\beta$ phase along the $\langle 110\rangle_{\beta}$ direction while the $\mathbf{b}$ and $\mathbf{c}$-axes lie along two $\langle 110\rangle_{\beta}$ directions of the $\beta$ phase perpendicular to the [100] direction and are equal to $\sqrt{2} a$, such that no lattice distortion or dilatation of the $\beta$ phase has been incorporated in the simulation. The lattice parameters used in simulation are measured from TEM diffraction, $\mathrm{a}=0.317 \mathrm{~nm}$, $\mathrm{b}=0.448 \mathrm{~nm}$ and $\mathrm{c}=0.448 \mathrm{~nm}$. The expected diffraction patterns from such a phase, overlaid on that expected from the $b c c$ matrix, in an orientation with respect to $\beta$ as described above, are given in Fig. 2(g) and 2(h) respectively. The simulated pattern in Fig. 2(g) may be compared with experimental pattern shown in Fig. 2(b), and that shown in Fig. 2(h) may be compared with the experimental pattern shown in Fig. 2(f). These comparisons reveal that, apart from the $b c c$ matrix and diffuse $\omega$ reflections, the diffraction maxima are consistent with the presence of the $\mathrm{O}^{\prime}$ phase.

The results confirm that $\mathrm{Zr}$ and $\mathrm{Al}$ additions to $\beta$-alloyed titanium reduce the stability of both the $\omega$ and the martensite phase $\left(\alpha^{\prime \prime}\right)$. The $\beta$ alloy compositions with $\mathrm{Zr}$ and $\mathrm{Al}$ additions were chosen such that the level of additions used would stabilize martensite at room temperature if the Bo-Md criterion, which has been experimentally validated for a large range of $\beta$ stabilizing additions, were to be followed. Al is an $\alpha$ stabilizer and should raise the $\mathrm{T}_{0}$ curve (at which the free energy of the $\alpha$ and $\beta$ phases are equal in the two-phase $\alpha+\beta$ region) and thus increase the Ms temperature. $\mathrm{Zr}$ is largely neutral with respect to $\alpha$ and $\beta$ phase stability, and should only mildly affect the $T_{0}$ curve. However, it appears inappropriate to regard $\mathrm{Al}$ and $\mathrm{Zr}$ as a ' $\beta$ stabilizer' in highly alloyed titanium, as has been suggested earlier [9, 10]. The present observations show that $\mathrm{Zr}$ and $\mathrm{Al}$ enhance the instability related to a $\{110\}<1 \overline{10}>$ shuffle, which is one component in the pathway of the $\beta \rightarrow \alpha$ transformation. The phonon mode associated with this displacement wave results in a displacement of atoms from the parent $\beta$ positions in $\langle 1 \overline{1} 0\rangle_{\beta}$ directions on every alternate $\{110\}_{\beta}$ plane. As a consequence, a structure with the $\mathrm{Cmcm}$ symmetry is formed that does not alter the parent $\beta$ lattice constants such that the new phase is completely coherent with $\beta$ matrix, and therefore forms as nano-domains in the parent matrix. This phase is responsible for the characteristic streaking and intensity maxima shown in Fig. 2(b), Fig. 2(d) and Fig. 3(b).

If the $\{110\}<1 \overline{1} 0>$ shuffle that results in the $\mathrm{O}^{\prime}$ phase precedes the $\{11 \overline{2}\}<111>$ shear that is associated with the Bain distortion of the parent $\beta$ to martensite, then it is likely that martensite formation will be suppressed since each martensite variant will allow only 2 of the 12 possible displacement directions associated with the $\{110\}<1 \overline{10}>$ shuffle, as shown in Fig. 1 . Thus, it is proposed that stability of the $\beta$ phase is determined by alloying effects on the relative softening of the elastic constants associated with the transformation each of the three phases that are observed, martensite ( $\alpha^{\prime}$ phase or $\alpha^{\prime \prime}$ phase), the $\omega$ phase and the $\mathrm{O}^{\prime}$ phase as described in Fig. 4. If the $\{11 \overline{2}\}<111>$ shear precedes the $\{110\}<1 \overline{10}>$ shuffle then martensite ( $\alpha^{\prime}$ phase or $\alpha^{\prime \prime}$ phase) results. When the latter shear mode precedes the former, nano-size $\mathrm{O}^{\prime}$ phase is produced. The $\mathrm{O}^{\prime}$ phase can coexist with $\omega$. If this shuffle instability occurs at higher temperatures, as with $\mathrm{Zr}$ addition, both the $\omega$ and martensite can be destabilized. The designation $\mathrm{O}^{\prime}$ has been used in this paper to 
imply that the path to this phase is composition invariant, and to distinguish it from the isothermal ordered orthorhombic phase, termed as $\mathrm{O}^{\prime \prime}$ phase, that has recently been reported during aging of $\beta$ alloys [11]. First principle calculations to evaluate the effect of additions on softening modes in the $\beta$ phase are currently in progress.

\section{Acknowledgment}

The support of this research by the National Science Foundation, under grant DMR1309270 , is very gratefully acknowledged. One of us, DB, gratefully acknowledges a visiting fellowship from the University of North Texas during which this research was initiated.

\section{References}

[1] W.G. Burgers, Physica., 1 (1934) 561-586.

[2] D. Banerjee, J.C. Williams, Acta Mater, 61 (2013) 844-879.

[3] M. Tahara, H.Y. Kim, T. Inamura, H. Hosoda, S. Miyazaki, Acta Mater., 59 (2011) 6208-6218.

[4] H.Y. Kim, L. Wei, S. Kobayashi, M. Tahara, S. Miyazaki, Acta Mater., 61 (2013) 4874-4886.

[5] S. Banerjee, P. Mukhopadhyay, Phase Transformations Examples from Titanium and Zirconium Alloys, Elsevier, 2007.

[6] A. Devaraj, S. Nag, R. Srinivasan, R.E.A. Williams, S. Banerjee, R. Banerjee, H.L. Fraser, Acta Mater., 60 (2012) 596-609.

[7] S. Nag, A. Devaraj, R. Srinivasan, R.E.A. Williams, N. Gupta, G.B. Viswanathan, J.S. Tiley, S. Banerjee, S.G. Srinivasan, H.L. Fraser, R. Banerjee, Phys. Rev. Lett., 106 (2011) 245701.

[8] J.C. Williams, B.S. Hickman, D.H. Leslie, Metall. Trans., 2 (1971) 477-484.

[9] M. Abdel-Hady, K. Hinoshita, M. Morinaga, Scripta Mater., 55 (2006) 477-480.

[10] S. Miyazaki, H.Y. Kim, H. Hosoda, T. Proceedings of the International Conference on Martensitic, Mater. Sci. \& Eng. A, 438-440 (2006) 18-24.

[11] Y. Zheng, R.E.A. Williams, H.L. Fraser, Scripta Mater., (Under Review).

\section{Figure Captions:}

Figure 1. Schematic representations of the three different instabilities in the $\beta$ phase in titanium alloys: (a) $\beta$ transforms to martensite by $\{11 \overline{2}\}<111>$ shear followed by a $\{110\}<1 \overline{1} 0>$ shuffle; (b) $\beta$ transforms to nano-domains $\left(\mathrm{O}^{\prime}\right.$ phase $)$ by a $\{110\}<1 \overline{10}>$ shuffle (atoms shuffle every other $\{110\}_{\beta}$ atomic plane); (c) $\beta$ transforms to $\omega$ phase by a longitudinal $2 / 3<111>$ displacement wave.

Figure 2. Diffraction patterns from $\beta$ quenched Ti-26Nb and Ti-26Nb-2Zr: (a) Part of the $[110]_{\beta}$ zone axis electron diffraction pattern from Ti-26Nb showing $\omega$ reflections (example shown by solid red arrow) and maxima at $1 / 2<112>_{\beta}$ positions (shown by the dashed white arrow); (b) Part of the $\langle 110\rangle_{\beta}$ zone axis diffraction pattern from Ti$26 \mathrm{Nb}-2 \mathrm{Zr}$ showing diffuse $\omega$ reflections with decreased intensity positions (again shown by the solid red arrow), and maxima at $1 / 2<112>_{\beta}$ positions of increased intensity (an example shown by the dashed white arrow) compared with these features in (a); (c) Part of the $[110]_{\beta}$ zone axis electron diffraction pattern from Ti-9Mo showing $\omega$ reflections (example shown by solid red arrow) and maxima at $1 / 2<112>_{\beta}$ positions (shown by the dashed white arrow); (d) part of the $\langle 110\rangle_{\beta}$ zone axis 
diffraction pattern from Ti-9Mo-9Al showing diffuse $\omega$ reflections with decreased intensity positions (again shown by the solid red arrow), and maxima at $1 / 2<112>_{\beta}$ positions of increased intensity (an example shown by the dashed white arrow) compared with these features in (c); (e) $[100]_{\beta}$ zone axis diffraction pattern from Ti$26 \mathrm{Nb}-2 \mathrm{Zr}$ showing streaking along $[110]_{\beta}$ directions along with intensity maxima in $1 / 2\{110\}_{\beta}$ (an example indicated by a solid white arrow) and $1 / 2\{310\}_{\beta}$ positions (an example indicated by a white circle); (f) A diffraction pattern from Ti-26Nb-2Zr with the electron beam tilted slightly from the $[100]_{\beta}$ zone axis showing the absence of intensity maxima in $1 / 2\{110\}_{\beta}$ and presence of intensity maxima in $1 / 2\{310\}_{\beta}$ positions; ( $\mathrm{g}$ ) simulated diffraction pattern of shuffled structure being overlaid on $[100]_{\beta}$ zone axis diffraction pattern; and, (h) simulated diffraction pattern of structure caused by the $\{110\}<1 \overline{1} 0>$ shuffle being overlaid on $[110]_{\beta}$ zone axis diffraction pattern. The indices given in $(\mathrm{g})$ and $(\mathrm{h})$ refer to the orthorhombic phase identified in this paper.

Figure 3. Conventional TEM image and Z-contrast HAADF-STEM images showing the co-existence of the $\omega$ phase and the nano-domains ( $\mathrm{O}^{\prime}$ phase): (a) dark field image formed using the intensity maxima in the $1 / 2\{310\}_{\beta}$ reflections in a selected area diffraction pattern with the beam parallel to a $\left\langle 110>_{\beta}\right.$ zone axis; (b) FFT of HAADFSTEM image showing the presence of spatial frequencies belonging to the $\omega$ phase (highlighted by red color solid circles) and also the nano-domains $\left(\mathrm{O}^{\prime}\right.$ phase, highlighted by blue color dashed circle); (c) High magnification HAADF-STEM image showing a nano- domain $\left(\mathrm{O}^{\prime}\right.$ phase, highlighted by the red dashed box); (d) A comparison of the arrangement of atomic columns (beam parallel to a $\langle 110\rangle_{\beta}$ zone axis) for the $b c c$ matrix and nano-domains $\left(\mathrm{O}^{\prime}\right.$ phase $)$.

Figure 4. Pseudo-binary phase diagrams showing the various instabilities in titanium alloy, without (a) and with (b) the $\{110\}<1 \overline{10} 0$ shear $\left(\mathrm{O}^{\prime}\right.$ phase $)$. 
Figure 1
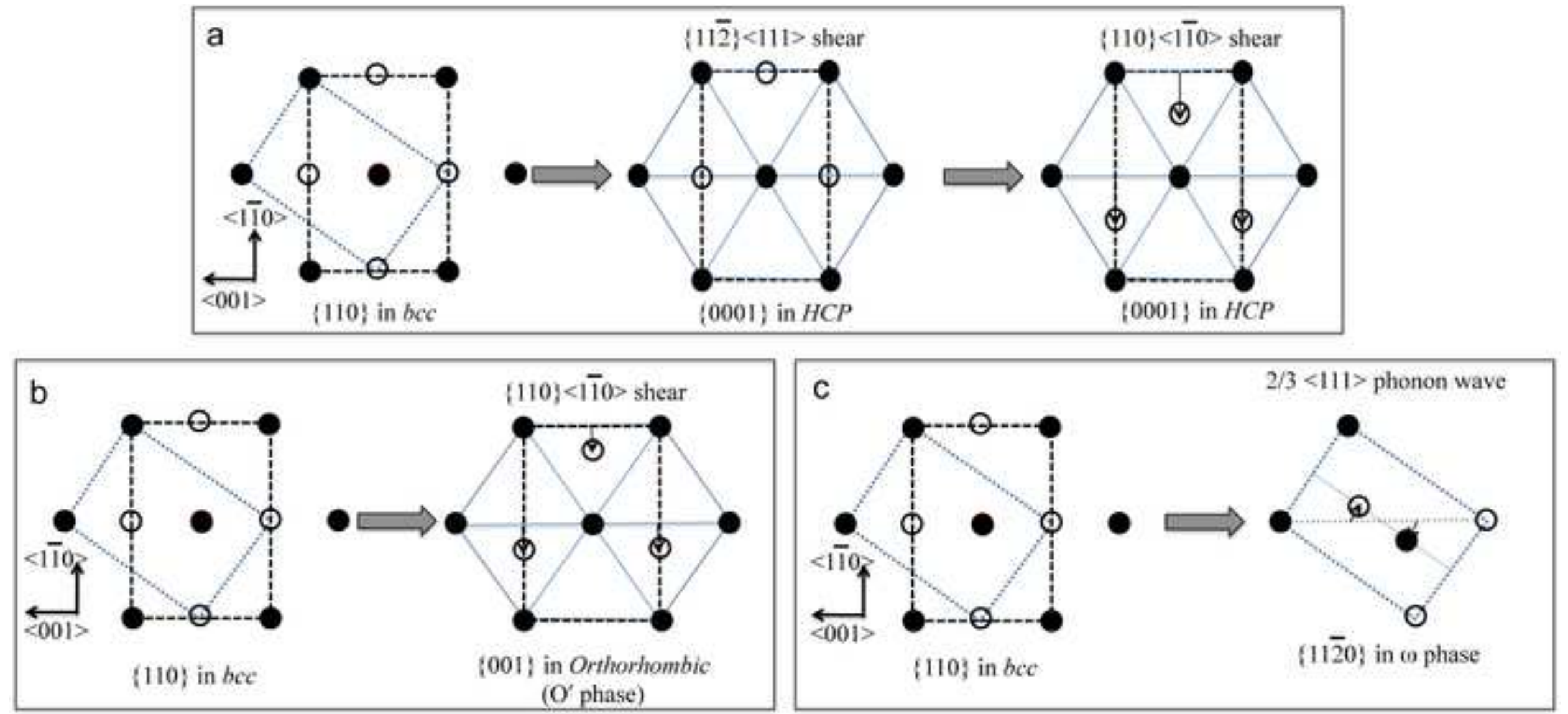


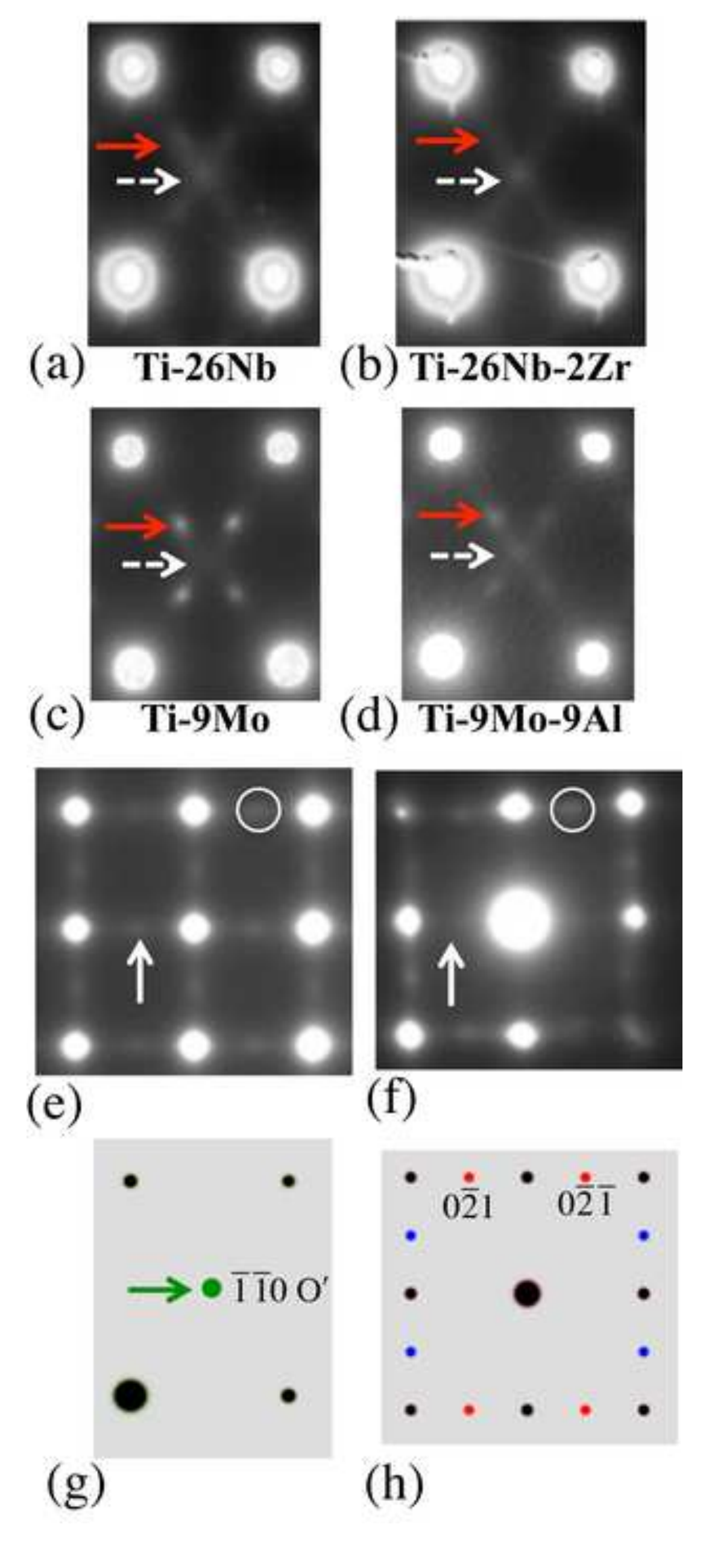

Figure 2

(b) Ti-26Nb-2Zr

(d) Ti-9Mo-9Al

(e)

(f)

(h)

$$
\text { - }
$$



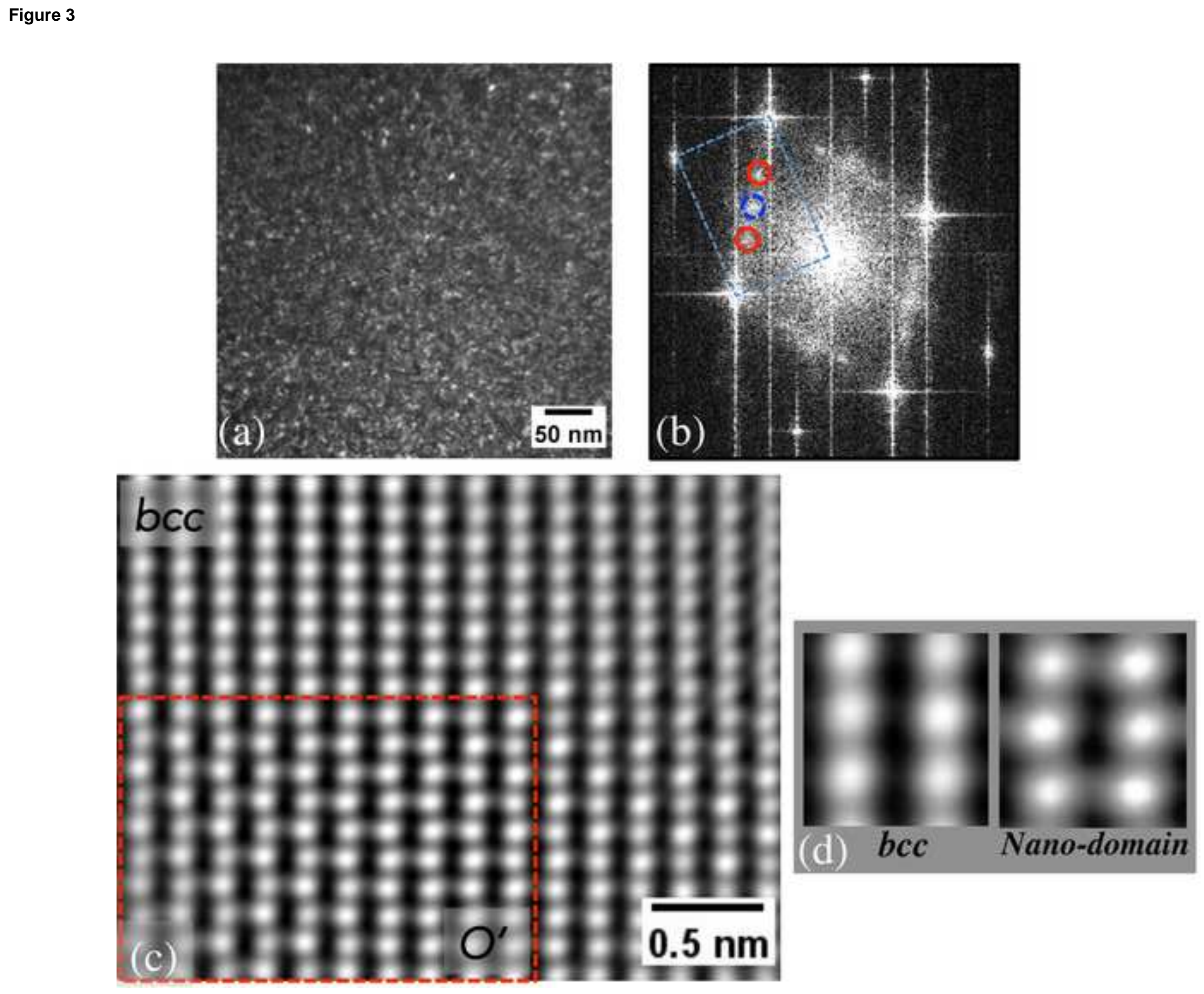

(d) bcc Nano-domain
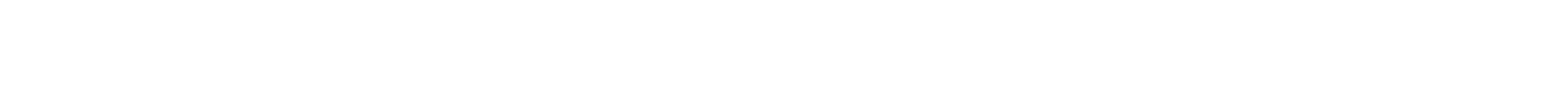

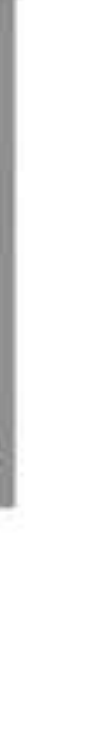

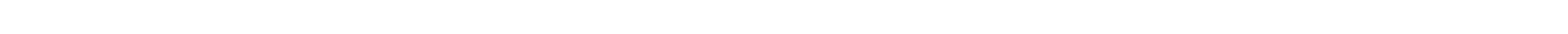

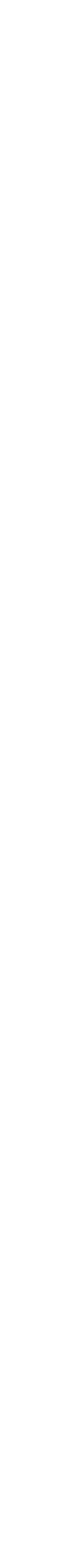



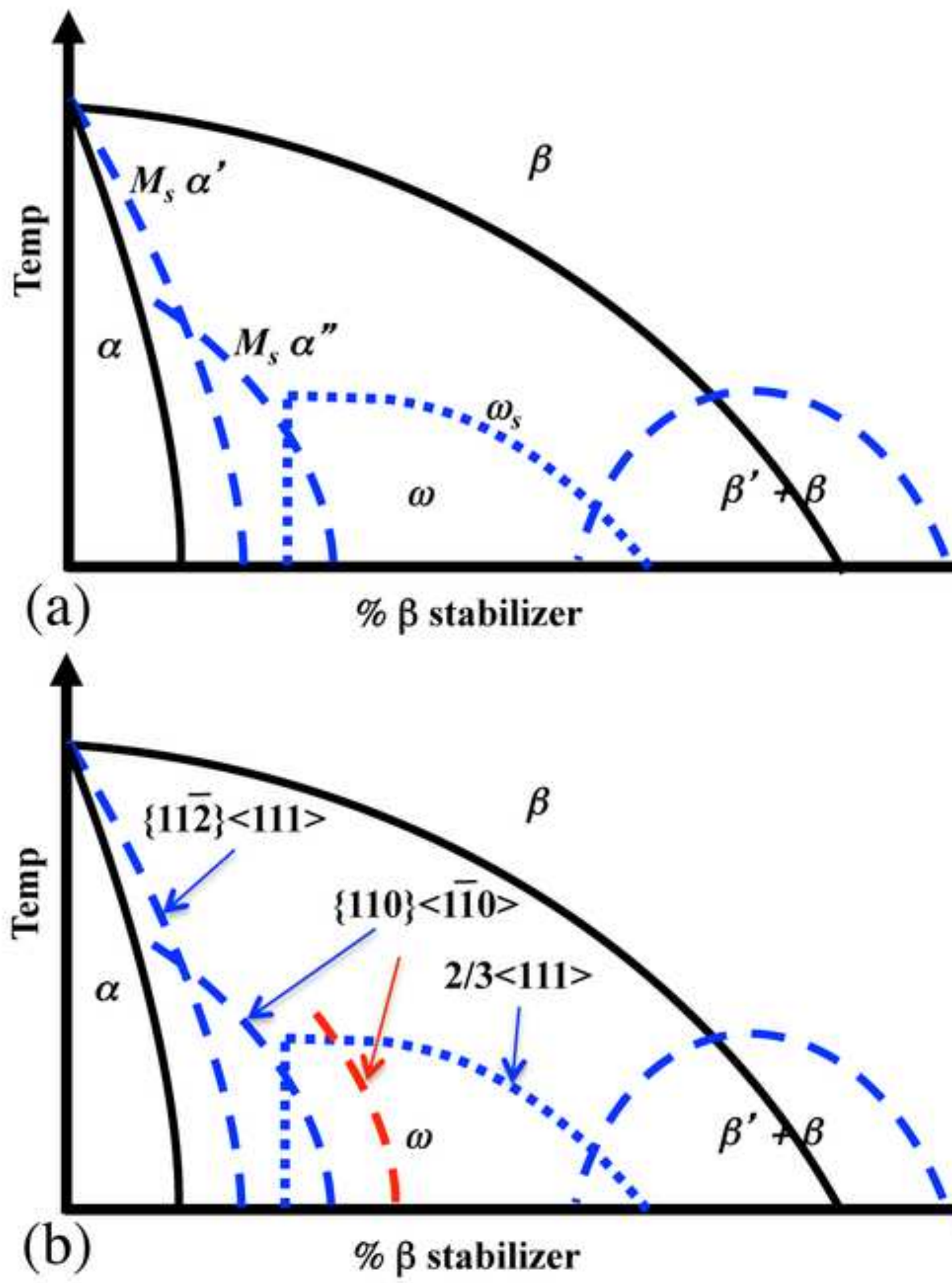

(b)

$\% \beta$ stabilizer 